\section{From the Residency Program D irector}

The goal of a Residency Program in Internal M edicine is to strive to train the next generation of practitioners and academicians in M edicine who will become physiciansscholars, scientists, teachers, and humanists.

We are excited that many of our residents are pursuing research opportunities in the laboratory or clinic. Such participation is a requirement for training at Jefferson. In addition, this journal serves as an outlet for scholarly work of a variety of types. We are pleased to support another issue of the Jefferson Forum to continue to highlight this work. This represents the sixth installment of the Jefferson Forum, which debuted in 1999. The journal is supported entirely through private contributions and unrestricted educational grants.
Subscriptions to the members of the D epartment of $M$ edicine are provided at no cost. The editors have maintained complete editorial independence to assemble and peer review the submissions for this installment.

The editors deserve our praise for a job well done. Please recognize that the creation of this journal is accomplished by a small team who still have to contend with the daily rigors of residency including night call, teaching their inpatient teams, long days, and preparing for boards. I thank them for extending Jefferson's tradition of excellence in education and enhancing the experience of our residents.

Gregory C. Kane M D

Associate Professor of M edicine

Residency Program Director

D epartment of M edicine

\title{
Welcome to the 6th Edition of the Jefferson M edicine Forum
}

We are very excited to bring you this new and improved volume of the Forum because it exhibits the talents of the Internal M edicine Residents. This edition showcases many of the residents' strengths in writing scholarly projects, creating research studies, and interpreting modern diagnostic imaging. Whether it is a discussion of an interesting case report or a perplexing el ectrocardiogram, all the work is truly original and academic. We applaud the contributing authors for all their creativity and dedication to excellence. All of these articles are the result of a lot of hard work in and out of the hospital. 